[2] Neel A., Henry B., Barbarot S. et al. Long-term effectiveness and safety of interleukin-1 receptor antagonist (anakinra) in Schnitzler syndrome: a french multicenter study Autoimmunity. Reviews. 2014;13:1035-1041

[3] Krause K., Tsianakas A.,Wagner N. et al. Efficacy and safety of canakinubab in Schnitzler syndrome: a multicenter randomized placebo-controlled study. 2017; J Allergy Clin Immunol 2017;139:1311-20.

Disclosure of Interests: None declared

DOI: 10.1136/annrheumdis-2019-eular.4611

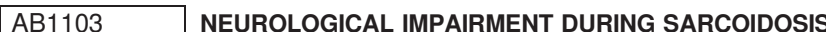

sameh sayhi ${ }^{1}$, Rim Dhahri ${ }^{1}$, Najah Boussetta ${ }^{1}$, Bilel Arfaoui ${ }^{1}$, Feida Laajili ${ }^{2}$, Bassem Louzir ${ }^{1}$, Hajer Derbali ${ }^{3}$, Linda Mrisa ${ }^{3}$, Ridha Mrisa ${ }^{3} .{ }^{1}$ Military Hospital of Tunis Tunisia, Internal Medicine, Tunis, Tunisia; ${ }^{2}$ Military Hospital of Tunis Tunisia, Autoimmune Diseases Unit Research UR17DN02, Tunis, Tunisia; ${ }^{3}$ Military Hospital of Tunis Tunisia, Neurology, Tunis, Tunisia

Background:

Objectives: To describe neurological impairment characteristics in sarcoidosis.

Methods: This was a descriptive and retrospective study including 65 patients with sarcoidosis, followed in the departments of internal medicine and neurology at the Military Hospital of Tunis over a period of 20 years from 1997 to 2017.

Results: A total of 65 patient files have been selected, of which 38 have neurological involvement.

Thirty-eight patients met the inclusion criteria for Neurosarcoidosis. According to Zajicek's criteria, the diagnosis of neurosarcoidosis was certain in 2 cases, probable in 18 cases and possible in 18 cases.

Neurological disorders were symptomatic in $58.5 \%$ of the studied population. Neurological signs were inaugural in 9 patients (14\% of cases). A central neurological involvement was demonstrated in 33 patients $(86.8 \%)$, the peripheral nervous system was affected in 5 patients $(13.1 \%)$, and cranial nerve involvement was found in 10 patients (26.3\%).\%). Ten patients have had both central and peripheral impairement.

Neurological involvement was significantly associated with cardiac, renal extra-thoracic, ophthalmologic, articular and cutaneous involvement ( $p$ $<0.05)$

Genotyping of the conversion enzyme and HLA typing did not show any particular pattern of neurosarcoidosis in comparison with patients without neurological signs.

Conclusion: Neurological impairement was frequently observed in our series. It was also significantly associated with multivisceral involvement without particular genetic pattern.

Disclosure of Interests: None declared

DOI: 10.1136/annrheumdis-2019-eular.7883

\section{AB1104 GENETIC ASPECTS OF SARCOIDOSIS IN A TUNISIAN POPULATION}

sameh sayhi ${ }^{1}$, Rim Dhahri ${ }^{1}$, Najah Boussetta ${ }^{1}$, Souha Hannachi ${ }^{1}$, Feida Laajili ${ }^{2}$, Bassem Louzir ${ }^{1}$, Hajer Derbali ${ }^{3}$, Linda Mrisa ${ }^{3}$, Ridha Mrisa ${ }^{3}{ }^{1}$ Military Hospital of $^{2}$ Tunis Tunisia, Internal Medicine, Tunis, Tunisia; ${ }^{2}$ Military Hospital of Tunis Tunisia, Autoimmune Diseases Unit Research UR17DN02, Tunis, Tunisia; ${ }^{3}$ Military Hospital of Tunis Tunisia, Neurology, Tunis, Tunisia

Background:

Objectives: To determine the frequencies of the HLA alleles and genotypes of the ACE gene.

Methods: This is a cross sectional study of 65 patients with sarcoidosis, followed in the departments of internal medicine and neurology at the Military Hospital of Tunis. The genetic study involved only 50 patients. DNA extraction was performed to determine the frequencies of the HLA alleles and genotypes of the ACE gene.

Results: 1. HLA typing

The analysis of HLA typing allowed us to estimate the different frequencies of alleles in patients. The calculation of the allelic frequency found that the most frequent alleles are HLA-DRB1 * 1501 alleles with a frequency of $38 \%$ and HLA-DRB1 * 0301 of $28 \%$. Other alleles are at lower frequencies (Table1).

\begin{tabular}{|c|c|c|}
\hline $\begin{array}{l}\mathrm{HLA} \\
\mathrm{N}=50 \\
\end{array}$ & $\mathbf{N}$ & $\%$ \\
\hline $\begin{array}{l}\text { HLA- } \\
\text { DRB1*1501 }\end{array}$ & 19 & $38 \%$ \\
\hline $\begin{array}{l}\text { HLA- } \\
\text { DRB }{ }^{*} 0301\end{array}$ & 14 & $28 \%$ \\
\hline $\begin{array}{l}\text { HLA- } \\
\text { DRB } 1 * 1106\end{array}$ & 9 & $18 \%$ \\
\hline $\begin{array}{l}\text { HLA- } \\
\text { DRB1 }{ }^{*} 0401\end{array}$ & 8 & $16 \%$ \\
\hline
\end{tabular}

2. Genotyping of the ECA gene

Genotyping of the polymorphism of the angiotensin converting enzyme (ACE) involved 50 patients. Genotypic frequencies (II, ID and DD) and allele frequencies ( $I$ and $D)$ were estimated in order to evaluate the frequencies of each genotype and the 2 alleles for this pathology. The genotypic and allelic frequency results were summarized in Table 2.

\begin{tabular}{lcc}
\hline Genotypes & $\mathbf{N}$ & $\%$ \\
II & 11 & $22 \%$ \\
ID & 24 & $48 \%$ \\
DD & 15 & $30 \%$ \\
Alleles & & \\
I & 46 & $46 \%$ \\
D & 54 & $54 \%$ \\
\hline
\end{tabular}

Conclusion: The most frequent alleles in Tunisian patients with sarcoidosis are HLA-DRB1 * 1501 alleles with a frequency of $38 \%$ and HLADRB1 * 0301 of $28 \%$. The genotypic and allelic results showed that genotype ID was the most frequent with $48 \%$ with a predominance of the $D$ allele in $54 \%$.

References:

\section{DISCLOSURE OF INTERESTS}

None declared

DOI: 10.1136/annrheumdis-2019-eular.7967

\section{AB1105 A NOVEL AUTOINFLAMMATORY AND LYMPHOPROLIFERATIVE SYNDROME ASSOCIATED WITH PIM1 MUTATIONS}

Giovanna Ferrara ${ }^{1}$, Silvio Polizzi ${ }^{2}$, Erica Valencic ${ }^{3}$, Annalisa Chiocchetti ${ }^{4}$, Josef Vuch ${ }^{3}$, Alessia Pin ${ }^{1}$, Elisa Piscianz ${ }^{1}$, Diego Vozzi ${ }^{3}$, Serena Pastore ${ }^{3}$, Paola Tomietto ${ }^{5}$, Andrea Taddio ${ }^{6}$, Flavio Faletra ${ }^{3}$, Umberto Dianzani ${ }^{4}$, Alberto Tommasini ${ }^{3} .{ }^{1}$ University of Trieste, Trieste, Italy; ${ }^{2}$ Meyer Children's Hospital, Trieste, Italy; ${ }^{3}$ Institute of Maternal and Child Health, IRCCS Burlo Garofolo, Trieste, Italy; ${ }^{4}$ Universita' del Piemonte Orientale, Novara, Italy; ${ }^{5}$ Azienda Sanitaria Universitaria Integrata Trieste, Cattinara Teaching Hospital, Trieste, Italy; ${ }^{6}$ Institute of Maternal and Child Health, IRCCS Burlo Garofolo and University of Trieste, Trieste, Italy

Background: Whole exome sequencing can allow genetic diagnosis in subjects with long lasting clinical stories not supporting any well-defined disorder.

A 35-year-old man was referred to ophthalmologist's evaluation for blurry vision in his left eye. The fundus examination showed choroidal lesions in both eyes. His past medical history was relevant for celiac disease, recurrent episodes of fever and skin rashes with leukocytoclastic vasculitis, inflammatory lesions of the osteoarticular and muscular system, one episode of aseptic meningitis, an intracranial granuloma and two episodes of anterior uveitis. He had also splenomegaly with non-caseating granulomas. Brain TC founded multiple lytic and sclerotic skull lesions. He was diagnosed with atypical sarcoidosis and treated with oral steroid and methotrexate.

Laboratory data always showed elevated erythrocyte sedimentation rate, strong positive C-reactive protein and polyclonal gammopathy.

Objectives: To describe functional and genetic data supporting the role of a PIM1 mutation in the multisystemic inflammation and lymphoproliferation of the patient.

Methods: Whole exome sequencing (WES) analysis. Flow-cytometry to evaluate Pim1 expression, Bad phosphorylation (target of Pim1 kinase) and the effect of PIM inhibitor on peripheral blood mononuclear cell (PBMC) viability. RNAseq was on primary fibroblasts from the patient and 